PROCEEDINGS OF THE

AMERICAN MATHEMATICAL SOCIETY

Volume 124, Number 11, November 1996

\title{
INDUCING CHARACTERS AND NILPOTENT SUBGROUPS
}

\author{
GABRIEL NAVARRO \\ (Communicated by Ronald M. Solomon)
}

\begin{abstract}
If $H$ is a subgroup of a finite group $G$ and $\gamma \in \operatorname{Irr}(H)$ induces irreducibly up to $G$, we prove that, under certain odd hypothesis, $\mathbf{F}(G) \mathbf{F}(H)$ is a nilpotent subgroup of $G$.
\end{abstract}

\section{INTRODUCTION}

If some character of a subgroup $H$ of a finite group $G$ induces irreducibly up to $G$, one expects $H$ to be large enough to contain nontrivial information on $G$. In this note, we relate the Fitting subgroups of $H$ and $G$.

Theorem A. Let $H \subseteq G$ and suppose that $\gamma$ is a character of $H$ with $\gamma^{G} \in \operatorname{Irr}(G)$. If either $|G: H|$ or $|H: \mathbf{F}(H)|$ is odd, then $\mathbf{F}(G) \mathbf{F}(H)$ is a nilpotent subgroup of $G$.

Notice that Theorem A is no longer true without the odd hypothesis, $G L(2,3)$ being a solvable counterexample (we may take $H$ to be a 3-Sylow normalizer).

Theorem A may be applied to study characters induced from nilpotent subgroups, and this is what we do in Section 3 below.

We thank M. Isaacs for many conversations on this subject and the University of Valencia for financial support while visiting the United States.

\section{Proof of Theorem A}

The proof of our Theorem A relies on the deep facts on odd fully ramified sections discovered by I. M. Isaacs ([1]).

(2.1) Theorem. Let $L \subseteq K \triangleleft G$ with $L \triangleleft G, K / L$ abelian, and assume that either $|G: K|$ or $|K: L|$ is odd. Let $\phi \in \operatorname{Irr}(L)$ be invariant in $G$ and assume that $\phi^{K}=e \theta$ for some $\theta \in \operatorname{Irr}(K)$ and integer $e$. Then there exists $U \subseteq G$ such that

(a) $U K=G$ and $U \cap K=L$,

(b) If $|K / L|>1$ and $\xi \in \operatorname{Irr}(U \mid \phi)$, then $\xi^{G}$ is reducible.

Proof. This is a well known consequence of Theorems (9.1) and (9.2) of [1]. If $\xi^{G}$ is irreducible, Theorem (9.2) forces the canonical character $\psi$ to be irreducible. However, $\psi \bar{\psi}$ is the permutation character of $G$ on $K / L$ (see the values of the character $\psi$ ), and since this action cannot be transitive, $[\psi, \psi]>1$, a contradiction.

Received by the editors April 15, 1995.

1991 Mathematics Subject Classification. Primary 20C15.

Research partially supported by DGICYT.

(C)1996 American Mathematical Society 
Proof of Theorem A. We argue by double induction, first on $|G|$ and second on $|G: H|$, and we assume that $H$ is proper in $G$.

First, we show that it is no loss to assume that $G=H \mathbf{F}(G)$. If we write $F=$ $\mathbf{F}(G)$ and $K=H F<G$, since $\gamma^{K}$ is irreducible, and either $|K: H|$ or $|H: \mathbf{F}(H)|$ is odd, by the inductive hypothesis, we have that $\mathbf{F}(K) \mathbf{F}(H)$ is nilpotent. But then, since $F \subseteq \mathbf{F}(K)$, the theorem follows in this case.

We claim that we may also assume that $H$ is a maximal subgroup of $G$. If $H<J<G$, arguing as before, we have that $(F \cap J) \mathbf{F}(H)$ is nilpotent. Since $J=(F \cap J) H$, we have that $(F \cap J) \mathbf{F}(H) \subseteq \mathbf{F}(J)$. Then, we have that either $|G: J|$ or $|J: \mathbf{F}(J)|$ is odd. Now, since $\left(\gamma^{J}\right)^{G}$ is irreducible, by induction on $|G: H|$, we have that $F \mathbf{F}(H) \subseteq F \mathbf{F}(J)$ is nilpotent, and this proves the claim.

Now, write $L=F \cap H$ and notice that $L$ is normal in $G$, because $L \triangleleft H$ and $L<\mathbf{N}_{F}(L)$. Since $H$ is maximal (and $F$ is nilpotent), we deduce that $F / L$ is an abelian $p$-chief factor of $G$, for some prime $p$.

Write $\mathbf{F}(H)=X \times Y$, where $X$ is the Sylow $p$-subgroup of $\mathbf{F}(H)$ and $Y$ its $p$-complement and similarly write $F=F_{p} \times F_{p^{\prime}}$. Since $F / L$ is a $p$-group, then $F_{p} L=F$ and thus $F_{p} H=G$. Hence, we have that $\mathbf{F}(H) / L$ is a $p^{\prime}$-group, because $F_{p} X$ is a $p$-group normalized by $H$ and then normal in $G$.

We now start looking at characters. If $\theta \in \operatorname{Irr}(X)$ is a constituent of $\gamma_{X}$, we claim that $\theta$ is $G$-invariant (recall that $X=F_{p} \cap L$ is normal in $G$ ). Certainly, $\theta$ is $Y$-invariant, since $[X, Y]=1$ and therefore, the inertia group $V$ of $\theta$ in $H$ contains $\mathbf{F}(H)$. Since $\theta$ lies under $\gamma$, by the Clifford Correspondence there is a character $\delta \in \operatorname{Irr}(V)$ such that $\delta^{H}=\gamma$. Therefore, $\delta^{G}$ is irreducible, and so it is $\delta^{F V}$. Now, if $V<H$, then $F V$ is proper in $G$ and we apply the inductive hypothesis to conclude that $\mathbf{F}(V) F$ is nilpotent. Since $\mathbf{F}(H) \subseteq \mathbf{F}(V)$, the theorem is proven in this case. So we assume that $\theta$ is $H$-invariant. Now, since $H$ is maximal in $G$, we have that either $\theta$ is $G$-invariant or its inertia group in $G$ is exactly $H$. So, if $\theta$ is not $G$-invariant, we have that $I_{F_{p}}(\theta)=H \cap F_{p}=X$ and thus, we deduce that $\theta^{F_{p}}$ is irreducible. We now prove that this implies $F \mathbf{F}(H)$ to be nilpotent. First, notice that the $p^{\prime}$-group $Y$ acts on the $p$-group $F_{p}$ in such a way that $X \subseteq \mathbf{C}_{F_{p}}(Y)$. Since $F_{p} / X$ is a chief factor of $G$, we have that $\mathbf{C}_{F_{p}}(Y)=X$ or $\left[F_{p}, Y\right]=1$. Since $F \mathbf{F}(H)=F_{p} Y$, we may assume that $\mathbf{C}_{F_{p}}(Y)=X$. Hence, $\mathbf{C}_{F_{p} / X}(Y)=1$. But then, the $Y$-invariant irreducible character $\theta^{F_{p}}$ restricted to $X$ has a unique $Y$ invariant irreducible constituent, by Problem (13.4) of [2], for instance. Since $X$ is centralized by $Y$, this implies that $\theta$ is stabilized by $F_{p}$. Since $\theta$ induces irreducibly up to $F_{p}$, by Problem (6.1) of [2], we have that $F_{p}=X$ and thus, that $G=H$, a contradiction. This proves $\theta$ to be $G$-invariant, as claimed.

Now, since $\left(\left|F Y: F_{p}\right|,\left|F_{p}: X\right|\right)=1$ and $\mathbf{C}_{F_{p} / X}(Y)=1$, we are in the hypotheses of Problem (13.10) of [2]. Hence, we may conclude that there exists a unique $Y$ invariant $\phi \in \operatorname{Irr}\left(F_{p}\right)$ lying over $\theta$. Since $Y \triangleleft H$, if $h \in H$, notice that $\phi^{h}$ is $Y$ invariant and lies over $\theta$. Therefore, by uniqueness, we have that $\phi$ is $G$-invariant. Hence, by Mackey, $\left(\gamma^{G}\right)_{F_{p}}$ is a multiple of $\phi$.

We are now ready to apply the Going Down Theorem (6.18) of [2] and we conclude that $\phi_{X}=\theta$ or that $\phi$ is fully ramified over $\theta$. In the first case by Corollary (4.2) of [3], we have that $\left(\gamma^{G}\right)_{H}$ is irreducible. This is impossible, unless $H=G$.

So, we may assume that $\phi$ is fully ramified over $\theta$. Now we wish to apply Theorem (2.1) and hence we check that $H$ (up to $G$-conjugacy) is the unique complement of $F_{p} / X$ in $G$. This follows by realizing that $H / X=\mathbf{N}_{G / X}(X Y / X)$ together with the 
fact that $X Y / X=(F Y \cap H) / X$ is a Hall $p$-complement of $F Y / X$. Now, since by our hypotheses we have that either $\left|F_{p}: X\right|$ or $|H: X|$ is odd, we apply Theorem (2.1) to get the final contradiction.

(2.3) Corollary. Suppose that $H \subseteq G$ is nilpotent. If $\gamma$ is a character of $H$ with $\gamma^{G}$ irreducible, then $\mathbf{F}(G) H$ is nilpotent.

Proof. In this case, $|H: \mathbf{F}(H)|$ is odd and Theorem A applies.

\section{Characters induced From nilpotent Subgroups}

In this section, we associate to any $\chi \in \operatorname{Irr}(G)$ a uniquely defined (up to conjugacy in $G$ ) pair $(S, \sigma)$, where $S$ is a subgroup of $G$ and $\sigma \in \operatorname{Irr}(S)$ induces $\chi$. For solvable groups, we will prove that $\chi$ is induced from a nilpotent subgroup if and only if $S$ is nilpotent. In other words, whenever $\chi \in \operatorname{Irr}(G)$ can be obtained via induction from a nilpotent subgroup, this can be done in a standard way.

Before introducing the pair $(S, \sigma)$ we need to derive a (perhaps) surprising consequence of (2.3).

(3.1) Theorem. Let $G$ be a solvable group and suppose that $\chi \in \operatorname{Irr}(G)$ is induced from a nilpotent subgroup. If $\chi_{\mathbf{F}(G)}$ is homogeneous, then $G$ is nilpotent.

Proof. Write $\chi=\gamma^{G}$, where $\gamma \in \operatorname{Irr}(H)$ and $H$ is maximal with respect to being nilpotent. By Corollary (2.3), we have that $F=\mathbf{F}(G) \subseteq H$. Now, let $M / F=$ $\mathbf{F}(G / F)$ and notice that $M \cap H=F$. This is because $F \subseteq M \cap H \subseteq M$ and hence, $M \cap H$ is both nilpotent and subnormal in $G$.

Write $\chi_{F}=e \theta$, where $\theta \in \operatorname{Irr}(F)$ and notice that, in the notation of [2], we have that $(G, F, \theta)$ is a character triple. By Theorem (11.28) of [2], we may find $\left(G^{*}, F^{*}, \theta^{*}\right)$ is an isomorphic character triple with $F^{*} \subseteq \mathbf{Z}\left(G^{*}\right)$. Therefore, observe that $M^{*}=\mathbf{F}\left(G^{*}\right)$ (we use the notation $(M / F)^{*}=M^{*} / F^{*}$, where ${ }^{*}$ also denotes the group isomorphism between $G / F$ and $\left.G^{*} / F^{*}\right)$. Since $H^{*}$ is also nilpotent and $\left(\gamma^{*}\right)^{G^{*}}$ is irreducible (because $\gamma^{G}$ is), we may again apply Corollary (2.3) to conclude that $M^{*} H^{*}$ is nilpotent. Hence, the group $M H / F$ is also nilpotent. But in this case, since $F \subseteq H$, we have that $H$ is nilpotent and subnormal in $M H$. Thus $H \subseteq \mathbf{F}(M H)$ and by the maximality of $H$, we conclude that $H=\mathbf{F}(M H) \triangleleft M H$. Now, since $M \cap H=F$, we have that $H / F \subseteq \mathbf{C}_{G / F}(M / F)$. Since $G$ is solvable, the group $M / F$ contains its own centralizer and thus, we have that $H / F \subseteq M / F$. This implies $H=F$ and $\theta=\gamma$. Now, since $\theta$ is $G$-invariant and induces irreducibly up to $G$, we conclude that $F=G$, as required.

If $G$ is a finite group and $\chi \in \operatorname{Irr}(G)$, we are going to define a uniquely determined (up to $G$-conjugacy) pair $(S, \sigma)$ associated to $\chi$, as follows. Choose $\theta \in \operatorname{Irr}(\mathbf{F}(G)$ ) to be any irreducible constituent of $\chi_{\mathbf{F}(G)}$, and let $\mu \in \operatorname{Irr}(T \mid \theta)$ be the Clifford correspondent of $\chi$ over $\theta$. If $T=G$, we define $(S, \sigma)=(G, \chi)$. On the other hand, if $T<G$, we inductively define $(S, \sigma)$ for $\chi$ to be the corresponding $(S, \sigma)$ for $\mu$.

Notice that $(S, \sigma)$ is determined up to conjugacy in $G$ and that it satisfies: $\mathbf{F}(G) \subseteq S, \sigma^{G}=\chi$ and $\sigma_{\mathbf{F}(S)}$ is homogeneous.

(3.2) Theorem. Let $G$ be a solvable group and let $\chi \in \operatorname{Irr}(G)$. Then $\chi$ is induced from a nilpotent subgroup if and only if $S$ is nilpotent.

Proof. Certainly, if $S$ is nilpotent, then $\chi$ is induced from a nilpotent subgroup so we prove the converse. We argue by induction on $|G|$. Let $F=\mathbf{F}(G)$, let $\theta \in \operatorname{Irr}(F)$ 
be under $\chi$ and let $\mu \in \operatorname{Irr}(T)$ be the Clifford correspondent of $\chi$ over $\theta$, so that the pair $(S, \sigma)$ for $\mu$ is also a pair for $\chi$.

Write $\chi=\gamma^{G}$, where $\gamma \in \operatorname{Irr}(H)$ and $H$ is nilpotent. By Corollary (2.3), we may assume that $F \subseteq H$ and by replacing $\theta$ by some $G$-conjugate, we may assume that $\gamma$ lies over $\theta$. Now, let $\tau \in \operatorname{Irr}(T \cap H \mid \theta)$ be the Clifford correspondent of $\gamma$ over $\theta$ and notice that $\tau^{G}=\chi$. By uniqueness of Clifford correspondents, we have that $\tau^{T}=\mu$. So we have that $\mu$ is also induced from a nilpotent subgroup. Therefore, if $T$ is proper in $G$, by induction, $S$ is nilpotent and we are done in this case. If $T=G$, by Theorem (3.1), $G$ is nilpotent and the result follows.

\section{REFERENCES}

1. I. M. Isaacs, Characters of Solvable and Symplectic Groups, Amer. J. Math. 95 (1973), 594635. MR 48:11270

2. I. M. Isaacs, Character Theory of Finite Groups, Academic Press, New York, 1976. MR $\mathbf{5 7 : 4 1 7}$

3. I. M. Isaacs, Characters of $\pi$-separable Groups, J. Algebra 86 (1984), 98-128. MR 85h:20012

Departament D’Algebra, Facultat de Matematiques, Universitat de Valencia, 46100 BurJassot, Valencia, Spain

E-mail address: gabriel@vm.ci.uv.es 